\title{
Addendum and Erratum: Nature of vibrational excitations in vitreous silica [Phys. Rev. B 56, 8605 (1997)]
}

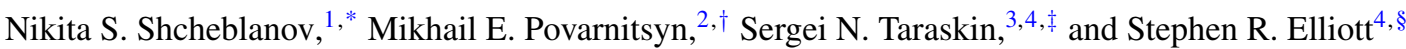 \\ ${ }^{1}$ Centre de Physique Théorique, CNRS, École Polytechnique, F-91128 Palaiseau, France \\ ${ }^{2}$ Joint Institute for High Temperatures, RAS, 13 Building 2 Izhorskaya street, Moscow 125412, Russia \\ ${ }^{3}$ St. Catharine's College, University of Cambridge, Cambridge CB2 1RL, United Kingdom \\ ${ }^{4}$ Department of Chemistry, University of Cambridge, Cambridge CB2 1EW, United Kingdom
}

(Received 9 August 2016; published 29 September 2016)

\begin{abstract}
The aim of this paper is to clarify details of the projectional analysis for atomic vibrations in vitreous silica presented in a paper by Taraskin and Elliott [Phys. Rev. B 56, 8605 (1997)]. The description of the stretching and bending modes has been discussed in detail while a description of the rotational modes and the procedure of their calculation have not been given in full detail. We specify the method for calculating the rotational modes omitted in the paper. We also correct a misprint in the expression for the $\mathbf{B}_{2}^{(E)}$ mode which, unfortunately, has been reproduced in the vibrational analysis of the $E$ mode in some publications.
\end{abstract}

DOI: 10.1103/PhysRevB.94.099903

The structure of vitreous $\mathrm{SiO}_{2}$ may be described as a network of corner-sharing $\mathrm{SiO}_{4}$ tetrahedra, $\mathrm{XY}_{4}$, or as being comprised of $\mathrm{Si}-\mathrm{O}-\mathrm{Si}$ or $\mathrm{O}-\mathrm{Si}-\mathrm{O}$ structural units, $\mathrm{XY}_{2}$. An ideal $\mathrm{XY}_{4}$ tetrahedron belongs to the cubic point group $T_{d}$ and its normal vibrational modes can be described in terms of symmetry coordinates transforming according to the irreducible representations of this group [1-3],

$$
3 F_{2}+A_{1}+E+F_{1} .
$$

Similarly, the atomic vibrations of the Si-O-Si or O-Si-O units can be decomposed into irreducible representations of the orthorhombic point group $C_{2 v}$ of a symmetric $\mathrm{XY}_{2}$ molecule [1-3],

$$
3 A_{1}+A_{2}+3 B_{1}+2 B_{2} .
$$

The symmetry modes $F_{2}$ (or $T_{2}$ in Ref. [4]), $A_{1}$, and $E$ for $\mathrm{SiO}_{4}$ structural units, and $A_{1}$ and $B_{1}$ modes for Si-O-Si and O-Si-O units have been discussed in detail in Ref. [5] while a description of the rotational modes $F_{1}$ and $B_{2}$ and the procedure for their calculation have not been given in full detail. Below, we specify the method for calculating the rotational modes omitted in Ref. [5]. For a perfect $\mathrm{SiO}_{4}$ structural unit, the rotations should be made around three fourfold rotation-reflection axes $\mathrm{S}_{4}$ passing through the central silicon atom, [1,6] as shown in Fig. 1(a) (the red line represents one of the axes). The direction vectors for the rotation axes, $\boldsymbol{w}_{k}(k=1,2,3)$, can be found in terms of position vectors of the oxygen atoms, $\mathbf{a}_{i^{\prime}}\left(i^{\prime}=1, \ldots, 4\right)$, as follows:

$$
\boldsymbol{w}_{1}=\mathbf{a}_{1}+\mathbf{a}_{2}, \boldsymbol{w}_{2}=\mathbf{a}_{1}+\mathbf{a}_{3}, \boldsymbol{w}_{3}=\mathbf{a}_{1}+\mathbf{a}_{4},
$$

with the origin of the coordinate system fixed at the $\mathrm{Si}$ atom [see Fig. 1(b)]. The choice of the rotation axes for a regular tetrahedron given by Eq. (3) is unique due to the property, $\sum_{i^{\prime}=1}^{4} \mathbf{a}_{i^{\prime}}=0$.

\footnotetext{
*nikita.shcheblanov@polytechnique.edu

†povar@ihed.ras.ru

${ }^{\ddagger}$ snt1000@cam.ac.uk

$\S_{\text {sre1@cam.ac.uk }}$
}

In vitreous silica, the $\mathrm{SiO}_{4}$ tetrahedra are distorted and this destroys the strict orthogonality of the rotation axes leading to some ambiguity in their choice. For example, axes (not necessarily mutually orthogonal) given by Eq. (3), in general, do not coincide with axes given by the same equations in which the vector $\mathbf{a}_{1}$ is replaced by $\mathbf{a}_{2}$ and $\mathbf{a}_{2}$ by $\mathbf{a}_{1}$. However, these distortions are not significant and average out when summing over many structural units, so that the sum of all contributions to the vibrational density of states (VDOS) corresponding to projections onto different symmetry modes including rotations practically coincide with the total VDOS obtained by numerical diagonalization of the dynamical matrix (see below).

In order to proceed with the projectional analysis, the symmetry displacement vector characterizing rotations of tetrahedra $\left(F_{1}\right)$ can be defined as:

$$
\mathbf{R}_{k}^{\left(F_{1}\right)}=\frac{1}{\sqrt{4}}\left\{\mathbf{0}, \hat{\mathbf{r}}_{1 k}, \hat{\mathbf{r}}_{2 k}, \hat{\mathbf{r}}_{3 k}, \hat{\mathbf{r}}_{4 k}\right\},
$$

where

$$
\hat{\mathbf{r}}_{i^{\prime} k}=\frac{\boldsymbol{w}_{k} \times \mathbf{a}_{i^{\prime}}}{\left|\boldsymbol{w}_{k} \times \mathbf{a}_{i^{\prime}}\right|} .
$$
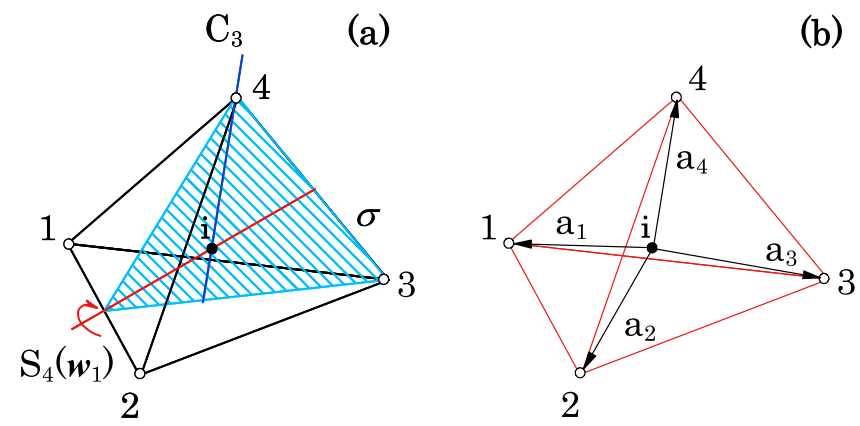

(b)

FIG. 1. Graphical representation of a regular tetrahedral $\mathrm{SiO}_{4}$ structural unit: (a) one of the rotation axes, $\mathrm{S}_{4}\left(\boldsymbol{w}_{1}\right)$ (shown in red) with $\boldsymbol{w}_{1}=\mathbf{a}_{1}+\mathbf{a}_{2}$, goes through the origin ( $\mathrm{Si}$ atom shown by the solid circle), $i$, connects two midpoints of edges 1-2 and 3-4 and thus belongs to the $\sigma$ reflection plane (shaded in blue) which also contains a threefold axis $\mathrm{C}_{3}$ [6]; (b) the position vectors $\mathbf{a}_{i^{\prime}}$ of the oxygen atoms (open circles). 


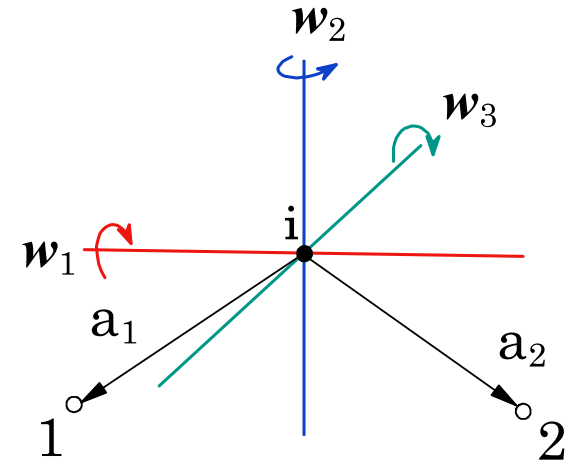

FIG. 2. Graphical representation of an $\mathrm{XY}_{2}$ structural unit. The direction vectors $\boldsymbol{w}_{k}$ for the rotation axes passing through the atom $\mathrm{X}$ (solid circle) are defined by the vector combinations [see Eq. (7)] of the position vectors $\mathbf{a}_{i^{\prime}}$ for the atoms Y (open circles).

Similarly, for rotations of Si-O-Si or O-Si-O structural units, the symmetry $\left(B_{2}\right)$ displacement vector can be defined as

$$
\mathbf{R}_{k}^{\left(B_{2}\right)}=\frac{1}{\sqrt{2}}\left\{\hat{\mathbf{r}}_{1 k}, \mathbf{0}, \hat{\mathbf{r}}_{2 k}\right\},
$$

where $\hat{\mathbf{r}}_{i^{\prime} k}$ are given by Eq. (5), and rotations are made around three orthogonal axes with the direction vectors

$$
\boldsymbol{w}_{1}=\mathbf{a}_{1}-\mathbf{a}_{2}, \boldsymbol{w}_{2}=\mathbf{a}_{1} \times \mathbf{a}_{2}, \boldsymbol{w}_{3}=\boldsymbol{w}_{1} \times \boldsymbol{w}_{2} .
$$

Here, the position vectors $\mathbf{a}_{i^{\prime}}$ point to atoms $\mathrm{Y}$ with atom $\mathrm{X}$ being at the origin (see Fig. 2).

Finally, the projection of the $j$ th displacement eigenvector $\mathbf{u}^{j}$ onto the rotation (around axis $\boldsymbol{w}_{k}$ ) displacement vector $\mathbf{R}_{k}$ $\left(\mathbf{R}_{k}^{\left(F_{1}\right)}\right.$ or $\left.\mathbf{R}_{k}^{\left(B_{2}\right)}\right)$ is given by Eq. (21) in Ref. [5] which for rotations reads

$$
r_{\mathbf{R}_{k}, i}^{j}=\sum_{i^{\prime}(i)} \mathbf{u}_{i^{\prime}}^{j} \cdot \mathbf{R}_{i^{\prime} k}
$$

where the summation is taken over all atoms $i^{\prime}$ of a particular structural unit $(i)$. The total contribution of the structural unit

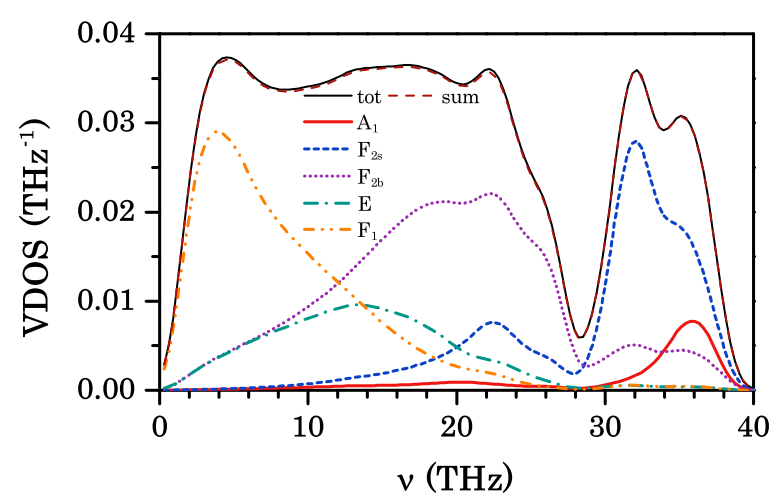

FIG. 3. The partial VDOS for the projections onto the vibrations of $\mathrm{SiO}_{4}$ structural units: $A_{1}$ symmetric stretching (solid red line), $F_{2 s}$ asymmetric stretching (dashed blue line), $F_{2 b}$ bending (dotted magenta line), $E$ bending (dot-dashed green line), $F_{1}$ solid-unit rotations (double dot-dashed orange line), and their sum (brown dashed line) coinciding with the total VDOS (black solid line). The results are obtained for a model with 8232 atoms [7].

(i) to the rotational motion equals the sum of the squares of projections onto three axes, i.e., $\sum_{k=1}^{3}\left(r_{\mathbf{R}_{k}, i}^{j}\right)^{2}$.

The expression for the $\mathbf{B}_{2}^{(E)}$ symmetry mode in Eq. (34) of the paper [5] contains a misprint. The correct expression reads

$$
\begin{aligned}
\mathbf{B}_{2}^{(E)}= & \mathcal{B}_{2}\left\{\mathbf{0},\left(\hat{\mathbf{b}}_{13}-\hat{\mathbf{b}}_{14}\right),\left(-\hat{\mathbf{b}}_{23}+\hat{\mathbf{b}}_{24}\right),\left(\hat{\mathbf{b}}_{31}-\hat{\mathbf{b}}_{32}\right),\right. \\
& \left.\left(-\hat{\mathbf{b}}_{41}+\hat{\mathbf{b}}_{42}\right)\right\} .
\end{aligned}
$$

In fact, the correct expression given by Eq. (9) has been used in the code for the calculation of the $E$ bending component, the results of which are presented in Fig. 15 of Ref. [5]. In order to confirm this, we have repeated the projectional analysis of Ref. [5] for a larger model (8232 atoms [7]) of vitreous silica constructed by using the BKS potential [8] (see Fig. 3). We should mention that, unfortunately, the misprint in the expression for the $\mathbf{B}_{2}^{(E)}$ mode has been reproduced in the VDOS analysis of the $E$ mode in some publications [9-12].
[1] G. Herzberg, Infrared and Raman Spectra of Polyatomic Molecules Molecular Spectra and Molecular Structure Vol. 2 (Van Nostrand, Reinhold, New York, 1945).

[2] G. Guimbretière, A. Bouchet, V. Rodriguez, M. Couzi, D. Talaga, T. Buffeteau, and L. Canioni, J. Phys. Chem. C 112, 17906 (2008).

[3] B. Hehlen and G. Simon, J. Raman Spectrosc. 43, 1941 (2012).

[4] A. Pasquarello, J. Sarnthein, and R. Car, Phys. Rev. B 57, 14133 (1998).

[5] S. N. Taraskin and S. R. Elliott, Phys. Rev. B 56, 8605 (1997).
[6] M. Hamermesh, Group Theory and its Application to Physical Problems (Addison-Wesley, Massachusetts, 1962).

[7] N. S. Shcheblanov and M. E. Povarnitsyn, Europhys. Lett. 114, 26004 (2016)

[8] A. Carré, L. Berthier, J. Horbach, S. Ispas, and W. Kob, J. Chem. Phys. 127, 114512 (2007).

[9] C. Oligschleger, Phys. Rev. B 60, 3182 (1999).

[10] A. B. Mukhopadhyay, C. Oligschleger, and M. Dolg, Phys. Rev. B 68, 024205 (2003).

[11] A. B. Mukhopadhyay, Ph.D. thesis, Universität zu Köln, 2004.

[12] A. B. Mukhopadhyay, C. Oligschleger, and M. Dolg, J. NonCryst. Solids 351, 1151 (2005). 